\title{
Carbon nano-cages created as cubes
}

W e have produced a new form of graphitic nano-cages, rectangular parallelepipeds or cubes, by arc evaporation of carbon with the alkaline-earth metals calcium or strontium. The cubes contain 5 to 20 layers of multiwalled graphitic carbon and have edges ranging in length from 20 to 100 nanometres. Their shape can be controlled by the type of metal catalyst selected.

The method used to obtain these cubes was the same as that for synthesizing carbon nanocapsules filled with rare-earth ${ }^{1,2}$ and iron-group metals ${ }^{3}$ : that is, evaporating a metal-loaded graphite rod (anode) in a helium atmosphere by a direct-current arc discharge. The metal-loaded anode was prepared by packing a hole (measuring $30 \mathrm{~mm}$ deep by $3.2 \mathrm{~mm}$ in diameter) drilled in a graphite rod (50 $\mathrm{mm}$ long by $6 \mathrm{~mm}$ diameter) with small pieces of calcium (purity 99\%) or strontium (purity 99\%). The graphite used for the rod was of $99.998 \%$ purity, as was that for the $13-\mathrm{mm}$ cathode. The helium (purity 99.999\%) introduced into the arc chamber had a pressure of typically 100 and 600 torr. Discharge current and voltage were $70 \mathrm{amp}$ and about $25 \mathrm{~V}$ respectively.

After arc evaporations using both calcium and strontium, we found, in the soot deposited on the cathode surfaces, abundant cubic cages of graphitic carbon (Fig. 1a). The cages range in size from 20 to $100 \mathrm{~nm}$ and consist of multiwalled graphitic carbon with a spacing of $0.34 \mathrm{~nm}$ (Fig. 1b). Adjacent graphitic layers were out of register, or turbostratic. At some of the cubes' corners, extrusion of graphitic layers can be seen, while breakages or smooth folding occurs at other corners (arrows in Fig. 1b). Most of the hollow cubes contain smaller cubes, which are also hollow.

Some other shapes of nano-cage fullerenes ${ }^{4}$ and nanotube tips ${ }^{5}$ - are closed by the introduction of 12 pentagons into the hexagon network. But in the case of a
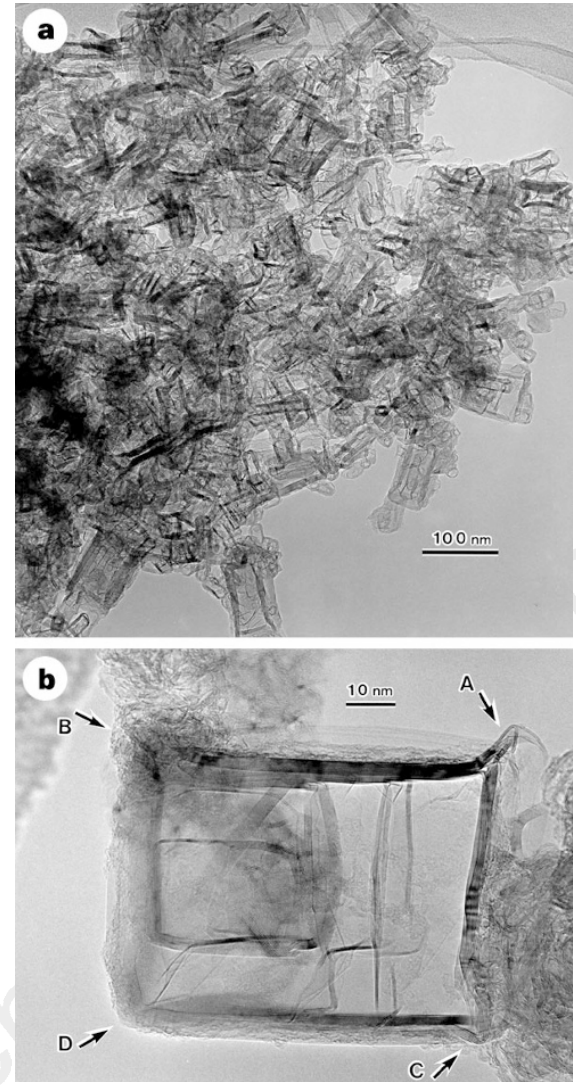

Figure 1 Transmission electron microscope images of hollow rectangular parallelepiped graphitic cages formed by the arc evaporation of a carbon/calcium composite. a, High-density aggregation of hollow rectangular parallelepipeds (low magnification). b, Aggregation of multiwalled graphitic carbon with a spacing of $0.34 \mathrm{~nm}$ (high resolution). At some corners, extrusion (arrows A, B) and breakage (arrow C) of graphitic layers can be seen, and sometimes graphitic layers are folded continuously (arrowD).

rectangular parallelepiped cage with eight vertices, pentagons or even four-membered rings cannot effect closure. Therefore, the rectangular corners suffer from geometrical frustrations, which may be relaxed by either extruding or discontinuing (breakage) graphitic layers at the corners.

The cubic shape is probably produced by folding graphitic sheets into a rectangular form, a process catalysed by alkalineearth metals. But questions remain over the detailed structures at the corners and the role of metallic atoms.

Although the hollow cubic cages predominated, we also found square cages filled with foreign materials. Most of the filling crystallites were carbides $\left(\mathrm{CaC}_{2}\right.$ and $\mathrm{SrC}_{2}$ ), but sometimes metallic strontium was encapsulated. Although these carbides and metal are hygroscopic, the crystallites nesting in the graphite cages did not degrade even when they were exposed to air. The closed graphitic cages effectively shield the inner materials from moisture, in a similar way to the carbon nanocapsules with polyhedral ${ }^{1,2}$ and spherical shapes ${ }^{3}$ that have been reported previously.

Graphitic carbon is found in forms and shapes as various as single-walled ${ }^{6,7}$ and multiwalled tubules ${ }^{8}$, cones $^{9}$, polyhedra $^{1,2}$ and spheres ${ }^{3,10}$. The topology of graphite networks governs the physical properties of nanometre-sized graphitic materials, so our success in producing a new form of graphitic cages should provide further opportunities for exploration of their exotic and unique properties.

Yahachi Saito, Takehisa Matsumoto

Department of Electrical and Electronic

Engineering, Mie University, Tsu 514, Japan

e-mail: saito@is.elec.mie-u.ac.jp

1. Tomita, M., Saito, Y. \& Hayashi, T. Jpn. J. Appl. Phys. 32, L280-L282 (1993).

2. Ruoff, R. S. et al. Science 259, 346-348 (1993).

3. Saito, Y. et al. J. Phys. Chem. Solids 54, 1849-1860 (1993).

4. Kroto, H. W., Heath, J. R., O'Brien, S. C., Curl, R. F. \& Smalley, R. E. Nature 318, 162-163 (1985).

5. Iijima, S., Ichihashi, T. \& Ando, Y. Nature 356, 776-778 (1992).

6. Iijima, S. \& Ichihashi, T. Nature 363, 603-605 (1993).

7. Bethune, D. S. et al. Nature 363, 605-607 (1993).

8. Iijima, S. Nature 354, 56-58 (1991).

9. Sattler, K. Carbon 33, 915-920 (1995).

10. Ugarte, D. Nature 359, 707-709 (1992).

\section{Tight knot values deviate from linear relations}

Applications of knots to the study of polymers have emphasized geometric measures on curves such as 'energy' 1 and 'rope length ${ }^{5-7}$, which, when minimized over different configurations of a knot, give computable knot invariants related to physical quantities $^{8}$. In DNA knots, electrophoretic mobility appears to be correlated with the average crossing number of rope-lengthminimizing configurations ${ }^{9}$, and a roughly linear empirical relation has been observed between the crossing number and rope length ${ }^{10}$. Here we show that a linear relation cannot hold in general, and we construct infinite families of knots whose rope length grows as the $3 / 4$ power of the crossing number $^{11}$. It can be shown that no smaller power is possible ${ }^{12-14}$.

One measure of geometric complexity for a space curve $\gamma$ is $A(\gamma)$, the average number of crossings in planar projections of $\gamma$. Another scale-invariant measure is rope length $L(\gamma)$, the quotient of arclength by thickness (the diameter of the largest uniform tube centred on $\gamma$ ).
If we fix a knot type $K$ (or a link type $K$ : our methods work for curves with one or many components), the infima (greatest lower bounds) of $A$ and $L$ over $\gamma$ in $K$ are the crossing number $C(K)$ and rope length $L(K)$ of $K$. A curve $\gamma$ achieving the infimum $L(K)$ is called 'tight'. Experiments ${ }^{9}$ with knotted DNA and simulations ${ }^{10}$ of thermal averages of such knots led us to seek mathematical relations between the invariants $C$ and $L$, and between $A$ and $L$ for tight curves.

For any fixed power $3 / 4 \leqslant p \leqslant 1$ we have constructed infinite families of knots and of links that have $L \sim C^{p}-$ the ratio $L / C^{p}$ is 


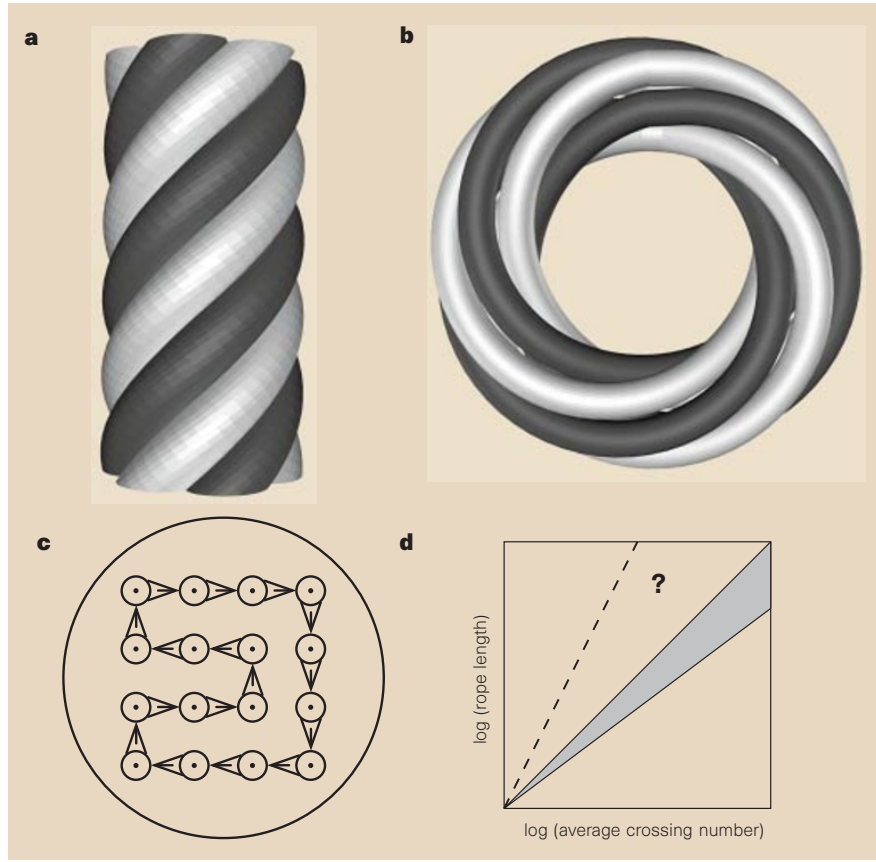

Figure 1 Construction for links with rope length $L$ growing as the $3 / 4$ power of crossing number $C$. a, This 'braid' has seven helical strands, one hidden in the centre. $b$, The braid can be bent into this Hopf link. c, The construction can be modified by following this circuit to produce a torus knot instead. d, The general relation between crossings and rope length when viewed at large scale: for any family of links, the growth rate must lie between powers $3 / 4$ and 2, and all powers below 1 are realized.

bounded above and below across each family. No $p<3 / 4$ can work, because $C \leqslant A$ and there is a universal lower bound ${ }^{12}$ for $L / A^{3 / 4}$. Furthermore, rope-length minimizers in a family with $L \sim C^{3 / 4}$ must satisfy $C \sim A$; thus these tight curves have $L \sim A^{3 / 4}$.

Any knot or link arises from a 'braid', a collection of $N$ ascending arcs in a cylinder $Z$, joining $N$ points on the bottom of $Z$ to the same $N$ points on the top. $Z$ is bent into a solid torus $S$, the top and bottom disks are identified, and the arcs are joined to give a link in $S$. For a braid with bounds on its slope, curvature and horizontal strand separation, this bending fixes rope length within a uniform factor (depending on the shape of $Z$ and $S$ ).

For a family of $\mathrm{N}$-component Hopf links with $L \sim C^{3 / 4}$, we took a cylinder $Z$ of height $h$ and radius $r$. $N$ points are distributed on the bottom disk of $Z$, and rotated one full turn as they ascend. The resulting braid (Fig. 1a) has $N$ helical strands of bounded slope and curvature, provided $h \approx r$; to separate strands, we needed $r \sim N^{1 / 2}$. Thus the braid (and the corresponding link, Fig. 1b) has rope length $L \sim h N \sim N^{3 / 2}$. As each component links every other component exactly once, $C \geqslant N(N-1)$; the standard projection has $N(N-1)$ crossings, so $C=N(N-1) \sim N^{2}$.

For $(N, N-1)$-torus knots in $S$ with $L \sim$ $C^{3 / 4}$, we repeated this construction in the lower half of $Z$. To define the rest of the braid, we chose a circuit joining the $N$ points (Fig. 1c) and slid each point along this circuit to the next while ascending through the upper half of $Z$. The horizontal distance a point travels is comparable to, at most, $r$. So, as before, we needed $h \sim r \sim N^{1 / 2}$ and rope length $L \sim N^{3 / 2}$. The minimum crossing number of this nnot $^{15}$ is $C=N(N-2) \sim N^{2}$. (Lattice knots with the same growth of crossing number have been found independently ${ }^{16}$.) Many further families of links can be thus constructed ${ }^{11}$.

If we plot $C$ versus $L$ on a $\log -\log$ scale (Fig. 1d), then links in any family with $L \sim C^{p}$ approach the ray of slope $p$. We have seen examples with $p=3 / 4$ (sublinear growth). To get $p=1$ (linear growth), consider simply linked chains of $N$ components, for which $L=2 \pi N+2(N-2)$, whereas $C=2(N-1)$ (ref. $11)$. Combining these examples yields families of links with any $3 / 4 \leqslant p \leqslant 1$.

It is unknown whether any family has superlinear growth. But if links with split, unknotted components are excluded, an argument based on embedding planar projections ${ }^{11}$ shows that no family has growth $p>2$.

\section{Jason Cantarella}

Department of Mathematics,

University of Pennsylvania,

Philadelphia, Pennsylvania 19104, USA

\section{Robert B. Kusner}

School of Mathematics, Institute for Advanced Study, Princeton, New Jersey 08540, USA

(Permanent address: Department of Mathematics,

University of Massachusetts, Amherst,

Massachusetts 01003, USA)

\section{John M. Sullivan}

Department of Mathematics,

University of Illinois, Urbana, Illinois 61801, USA e-mail:jms@math.uiuc.edu

1. O'Hara, J. Topology 30, 241-247 (1991).

2. Freedman, M. H., He, Z.-X., \& Wang, Z. Ann. Math. 139, 1-50 (1994).

3. Kim, D. \& Kusner, R. Exp. Math. 2, 1-9 (1993).

4. Kusner, R. B. \& Sullivan, J. M. in Geometric Topology (ed. Kazez, W. H.) 570-604 (Am. Math. Soc./Int. Press, Providence, 1997). 5. Kusner, R. B. \& Sullivan, J. M. in Topology and Geometry in

Polymer Science (eds Whittington, S., Sumners, D. \& Lodge, T.) 67-78 (Springer, New York, 1998).

6. Litherland, R. A., Simon, J., Durumeric, O. \& Rawdon, E. Topol. Appl. (in the press).

7. Nabutovsky, A. Comm. Pure Appl. Math. 48, 381-428 (1995)

8. Moffatt, H. K. Nature 384, 114 (1996).

9. Stasiak, A., Katritch, V., Bednar, J., Michoud, D. \& Dubochet, J. Nature 384, 122 (1996).
10. Katritch, V. et al. Nature 384, 142-145 (1996)

1. Cantarella, J., Kusner, R. B. \& Sullivan, J. M. (Preprint, Dept of Mathematics, Univ. of Illinois, 1997).

12. Cantarella, J., DeTurck, D. \& Gluck, H. (Preprint, Dept of Mathematics, Univ. of Pennsylvania, 1997).

13. Freedman, M. H. \& He, Z.-X. Ann. Math. 134, 189-229 (1991). 14. Buck, G. \& Simon, J. Topol. Appl. (in the press).

15. Murasagi, K. Trans. Am. Math. Soc. 237-260 (1991).

16. Diao, Y. \& Ernst, C. Topol. Appl. (in the press).

\section{Four-thirds power law for knots and links}

Physical knot theory has recently been applied to polymer dynamics, and specifically to gel electrophoresis of $\mathrm{DNA}^{1,2}$. Knot energies $^{3-6}$ measure the complexity of a knot conformation; minimum energy conformations are considered canonical or 'ideal' conformations. The rope length of a knot is one such measure of energy ${ }^{6}$, and an approximately linear relationship between rope length and the average crossing number for minimum rope-length conformations of simple knots has been reported ${ }^{7}$. Here I show that a linear relationship cannot hold in general: the rope length required to tie an $\mathrm{N}$-crossing knot or link varies at least between $\sim N^{3 / 4}$ and $\sim N$.

Consider four measures of knot conformation complexity. Imagine a solid disc of radius $R$ centred at $x$ and normal to $K$ at each point $x$ along the parametrized smooth knot $K . R(K)$ is the largest $R$ so that the disks are disjointed. The rope length is $L(K)=[\operatorname{arclength}(K)] /[R(K)]$. The crossing number $C(K)$ of the knot-type $\mathrm{K}$ is the necessary number of crossings in the planar diagram of $K$.

For points $x, y$ on $K$, let $\rho$ denote $|x-y|, \mathbf{r}$ the vector $(x-y) /|x-y|$, and $\mathrm{dx}$ a line element of $K$. The average crossing number is given by

$$
A(K)=(1 / 4 \pi) \iint_{K \times K}([\mathrm{~d} \mathbf{x}, \mathrm{dy}, \mathbf{r}] \mid) / \rho^{2}
$$

where the integrand numerator is the positive triple scalar product of the three vectors. This integral gives the average number of crossings of $K$, when viewed from an arbitrary perspective. The symmetric ener$\mathrm{gy}^{3}$ is given by

$$
S(K)=\iint_{K \times K}(|\mathrm{~d} \mathbf{x} \times \mathbf{r}||\mathrm{d} \mathbf{y} \times \mathbf{r}|) / \rho^{2}
$$

If $K$ is the centre curve of a radiating tube, $T$, with relatively small radius, which is relatively far from self-intersection, then $S(K)$ measures the self-radiation of the tube.

A natural $4 / 3$ power law relates rope length to measures based on inverse-square laws (including the crossing and writhe numbers, and $S(K)$ ), and a linear bound relates $S(K)$ to $A$. We can show ${ }^{4,17}$ that

$$
11 L(K)^{4 / 3} \geqslant S(K) \geqslant 4 \pi A(K) \geqslant C(K)
$$

Imagine a very long piece of rope packed as tightly as possible in a roughly spherical 